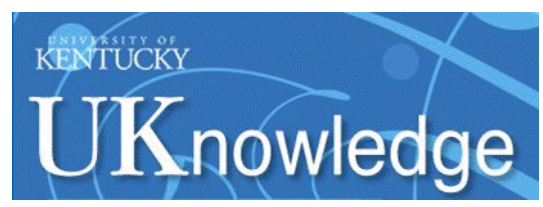

University of Kentucky

UKnowledge

\title{
Magnetic Response of Aperiodic Wire Networks Based on Fibonacci Distortions of Square Antidot Lattices
}

\author{
Barry Farmer \\ University of Kentucky, barry.farmer@uky.edu \\ Vinayak Bhat \\ University of Kentucky, vinayak8582@gmail.com \\ J. Sklenar \\ Northwestern University \\ Eric Teipel \\ University of Kentucky, epte223@G.uky.edu \\ Justin Woods \\ University of Kentucky, justin.s.woods@uky.edu
}

See next page for additional authors

Follow this and additional works at: https://uknowledge.uky.edu/physastron_facpub

Part of the Astrophysics and Astronomy Commons, Computer Engineering Commons, and the

Physics Commons

Right click to open a feedback form in a new tab to let us know how this document benefits you.

\section{Repository Citation}

Farmer, Barry; Bhat, Vinayak; Sklenar, J.; Teipel, Eric; Woods, Justin; Ketterson, J. B.; Hastings, J. Todd; and DeLong, Lance, "Magnetic Response of Aperiodic Wire Networks Based on Fibonacci Distortions of Square Antidot Lattices" (2015). Physics and Astronomy Faculty Publications. 247. https://uknowledge.uky.edu/physastron_facpub/247

This Article is brought to you for free and open access by the Physics and Astronomy at UKnowledge. It has been accepted for inclusion in Physics and Astronomy Faculty Publications by an authorized administrator of UKnowledge. For more information, please contact UKnowledge@lsv.uky.edu. 


\section{Magnetic Response of Aperiodic Wire Networks Based on Fibonacci Distortions of Square Antidot Lattices}

Digital Object Identifier (DOI)

http://dx.doi.org/10.1063/1.4913820

\section{Notes/Citation Information}

Published in Journal of Applied Physics, v. 117, no. 17, article 17B714, p. 1-3.

Copyright 2015 American Institute of Physics. This article may be downloaded for personal use only. Any other use requires prior permission of the author and the American Institute of Physics.

The following article appeared in Journal of Applied Physics, v. 117, no. 17, article 17B714, p. 1-3 and may be found at http://dx.doi.org/10.1063/1.4913820.

Authors

Barry Farmer, Vinayak Bhat, J. Sklenar, Eric Teipel, Justin Woods, J. B. Ketterson, J. Todd Hastings, and Lance DeLong 


\title{
Magnetic response of aperiodic wire networks based on Fibonacci distortions of square antidot lattices
}

\author{
B. Farmer, ${ }^{1}$ V. S. Bhat, ${ }^{1}$ J. Sklenar, ${ }^{2}$ E. Teipel, ${ }^{1}$ J. Woods, ${ }^{1}$ J. B. Ketterson, ${ }^{2}$ J. T. Hastings, ${ }^{3}$ \\ and L. E. De Long ${ }^{1, a)}$ \\ ${ }^{1}$ Department of Physics and Astronomy, University of Kentucky, Lexington, Kentucky 40506, USA \\ ${ }^{2}$ Department of Physics and Astronomy, Northwestern University, Evanston, Illinois 60208, USA \\ ${ }^{3}$ Department of Electric and Computer Engineering, University of Kentucky, Lexington, Kentucky 40506, USA
}

(Presented 5 November 2014; received 22 September 2014; accepted 27 October 2014; published online 5 March 2015)

\begin{abstract}
The static and dynamic magnetic responses of patterned ferromagnetic thin films are uniquely altered in the case of aperiodic patterns that retain long-range order (e.g., quasicrystals). We have fabricated permalloy wire networks based on periodic square antidot lattices (ADLs) distorted according to an aperiodic Fibonacci sequence applied to two lattice translations, $d_{l}=1618 \mathrm{~nm}$ and $d_{2}=1000 \mathrm{~nm}$. The wire segment thickness is fixed at $t=25 \mathrm{~nm}$, and the width $W$ varies from 80 to $510 \mathrm{~nm}$. We measured the DC magnetization between room temperature and $5 \mathrm{~K}$. Roomtemperature, narrow-band $(9.7 \mathrm{GHz})$ ferromagnetic resonance (FMR) spectra were acquired for various directions of applied magnetic field. The DC magnetization curves exhibited pronounced step anomalies and plateaus that signal flux closure states. Although the Fibonacci distortion breaks the fourfold symmetry of a finite periodic square ADL, the FMR data exhibit fourfold rotational symmetry with respect to the applied DC magnetic field direction. (C) 2015 AIP Publishing LLC.

[http://dx.doi.org/10.1063/1.4913820]
\end{abstract}

Advances in submicron-scale lithography make it possible to pattern ferromagnetic (FM) films into wire segments whose high shape anisotropy and narrow width $W$ strongly pin domain walls (DWs). ${ }^{1,2}$ Consequently, FM films patterned into periodic wire networks or antidot lattices (ADLs) can be engineered to control spin wave propagation and lowfield reversal. ${ }^{3,4}$ The bipolar "Ising" character of the magnetizations of narrow sub-micron segments in wire networks also permits controlled studies of frustration on cooperative behavior of artificial spin ices (ASI). ${ }^{5}$

Early ferromagnetic resonance (FMR) studies ${ }^{6,7}$ of periodic lattices of submicron permalloy rings and dots demonstrated the high sensitivity of spectra to the separation, edge imperfections, and small asymmetries of pattern features. Recent work implies low-field hysteresis, and spin wave propagation and localization are strongly affected by disorder in patterned FM films. ${ }^{3,4,8,9}$ On the other hand, all pattern vertices have very similar local environments in large-area periodic wire networks, which implies the order of segment switching in the reversal process is stochastic, and difficult to control. ${ }^{8}$ It is therefore important to investigate effects of controlled pattern disorder on the stability and dynamics of DW, reversal, and spin wave propagation.

We are motivated by a recent study of $2 \mathrm{D}$ artificial quasicrystals based on the Penrose P2 tiling (P2T) that has aperiodic translational symmetry combined with unusual fivefold rotational symmetry. Since quasicrystal patterns are determined by precisely defined algorithms, they are not amorphous, but long-range ordered, ${ }^{10}$ and they provide an interesting

\footnotetext{
${ }^{\text {a) }}$ Author to whom correspondence should be addressed. Electronic mail: delong@pa.uky.edu
}

paradigm for "intermediate order."11 Numerical simulations, DC magnetization data, and FMR spectra of P2T patterned into permalloy thin films reveal highly controlled magnetization reversal and mode symmetries. ${ }^{8}$ This is surprising, given local switching events in aperiodic P2T are mediated by a large number of inequivalent pattern vertices having asymmetric two-, three-, four-, and five-fold coordinations. ${ }^{9}$

Quasicrystal tilings are topologically related to a Fibonacci sequence ${ }^{12,13}$ that underpins an algorithm for constructing a one-dimensional lattice of long and short segment lengths, $d_{1}$ and $d_{2}$. The 1D Fibonacci lattice is long-range-ordered, but aperiodic, and can be viewed as a continuous distortion of a periodic lattice (i.e., $d_{1}=d_{2}$ ). However, the aperiodic, fivefold symmetry of the P2T cannot be obtained from a continuous distortion of a periodic lattice in two dimensions. Fibonacci lattices therefore form a novel class of thin-film FM metamaterials whose aperiodic, long-range order can be continuously varied in ways that are inaccessible to artificial quasicrystals with "forbidden" (fivefold) symmetries. ${ }^{12}$

Two-dimensional, multiply-connected wire networks of permalloy were fabricated on square Fibonacci lattices $(\mathrm{SFLs})^{12}$ using electron beam lithography and lift-off techniques (see Fig. 1). A continuous distortion $\left(d_{1}>d_{2}\right)$ is applied along both orthogonal primitive vectors of a periodic square lattice. In the present study, the lengths of long $\left(d_{1}\right)$ and short $\left(d_{2}\right)$ segments were kept constant at $1618 \mathrm{~nm}$ and $1000 \mathrm{~nm}$, respectively, but the segment width $(W)$ was varied (see Table I) to cover a range of stability for DW occupying the interior of film segments (see Fig. 3). A permalloy film of thickness $25 \mathrm{~nm}$ and $2 \times 2 \mathrm{~mm}^{2}$ overall dimension was deposited using electron beam evaporation, with a base pressure of $10^{-7}$ Torr. 

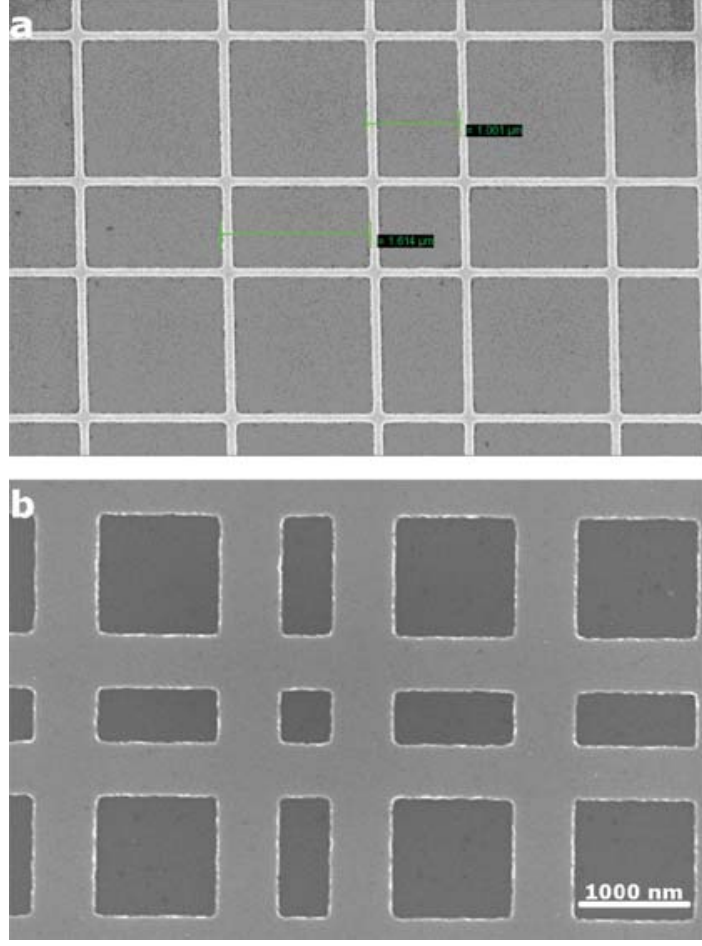

FIG. 1. (a) Distorted, aperiodic SFL sample III136A with long and short lattice spacings (corresponding to scale bars) $d_{1}=1618 \mathrm{~nm}$ and $d_{2}=1000 \mathrm{~nm}$, and segment width $W=80 \mathrm{~nm}$. Light color is Py; dark color is Si substrate. (b) SEM image of aperiodic Fibonacci lattice sample III135B composed of long and short lattice spacings $d_{1}=1618 \mathrm{~nm}$ and $d_{2}=1000 \mathrm{~nm}$, and segment width $W=510 \mathrm{~nm}$. Light color is Py; dark color is Si substrate. Scale bar indicates $1000 \mathrm{~nm}$.

DC magnetization data were acquired at room temperature with a Quantum Design MPMS5 SQUID Magnetometer in DC applied fields in the range $H= \pm 50 \mathrm{kOe}$. Roomtemperature, narrow-band (NB) FMR measurements were carried out at frequency $f=9.7 \mathrm{GHz}$ using a Bruker ESP 300E EPR Spectrometer in applied magnetic fields $H \leq 10 \mathrm{kOe}$.

The measured field dependences of the DC magnetization $M(H)$ of samples having different segment widths $W$ are compared in Fig. 2 for temperature $T=310 \mathrm{~K}$ and applied field $\boldsymbol{H}$ along a primitive ${ }^{12}$ SFL direction (horizontal axis in Fig. 1). Numerical simulations ${ }^{14}$ of the magnetization for $T=0$ indicate that domain walls occupy the interior of film segments (non-Ising behavior) for samples III135A and III135B, as shown in Fig. 3. Large "knees" at strong drops in

TABLE I. Square Fibonacci lattice parameters.

\begin{tabular}{lccc}
\hline \hline Sample number & Segment dimensions & $\mathrm{H}_{\mathrm{C}}(\mathrm{Oe})$ & Segment width \\
\hline III135A & $d_{1}=1618 \mathrm{~nm}$ & 130 & $W=350 \mathrm{~nm}$ \\
& $d_{2}=1000 \mathrm{~nm}$ & & \\
III135B & $d_{1}=1618 \mathrm{~nm}$ & 70 & $W=510 \mathrm{~nm}$ \\
& $d_{2}=1000 \mathrm{~nm}$ & & \\
III135E & $d_{l}=1618 \mathrm{~nm}$ & 230 & $W=150 \mathrm{~nm}$ \\
& $d_{2}=1000 \mathrm{~nm}$ & & \\
III136A & $d_{l}=1618 \mathrm{~nm}$ & 220 & $W=80 \mathrm{~nm}$ \\
& $d_{2}=1000 \mathrm{~nm}$ & & \\
\hline \hline
\end{tabular}

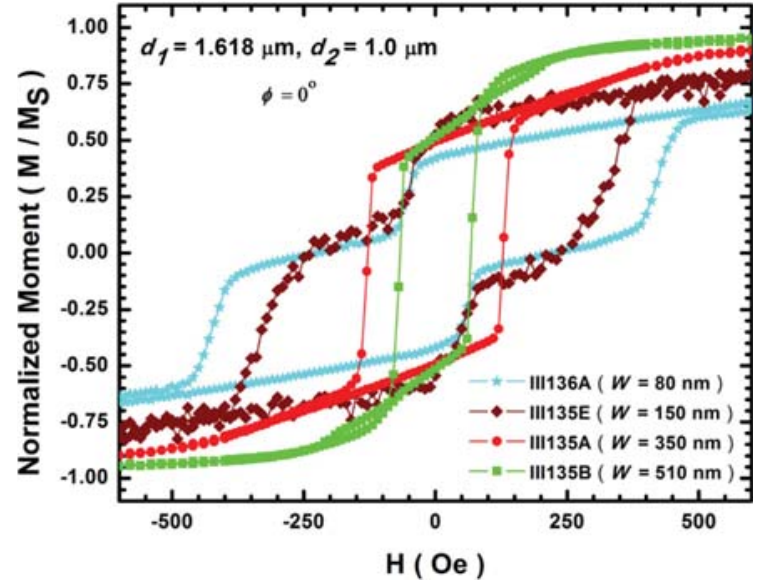

FIG. 2. Magnetization hysteresis $M(H)$ for angle $\phi=0^{\circ}$ between applied magnetic field $\boldsymbol{H}$ and the [10] SFL direction for several samples having different segment widths $W$. (The increased scatter for sample III135E data is due to the DC (rather than RSO) scan method used in SQUID magnetometer experiments.)

$M(H)$ are prominent features of data in Fig. 2 for samples with larger segment widths. Near-horizontal plateaus extending over wide (e.g., 340 Oe and 150 Oe for samples III136A and III135E, respectively) field intervals in the middle of the reversal regime are striking features of the hysteresis loops of samples with the smallest segment widths, which simulations show promote Ising behavior.

Previous data ${ }^{3,15}$ for the hysteresis loops of periodic square permalloy ADL with segment widths $W \geq 150 \mathrm{~nm}$ did not exhibit low-field plateaus. Small knee anomalies in $M(H)$ were previously ascribed to the switching of individual or small groups of film segments in simulations of P2T; ${ }^{9}$ whereas large drops in magnetization were associated with the generation of closed dipole loops ("vortices") formed by near-neighbor segments. ${ }^{8}$

Reduced temperature increases the coercive field of sample III136A, as shown in Fig. 4. The $T=5 \mathrm{~K}$ data exhibit a clear asymmetry that is commonly attributed to an exchange bias effect, which implies a magnetically hard

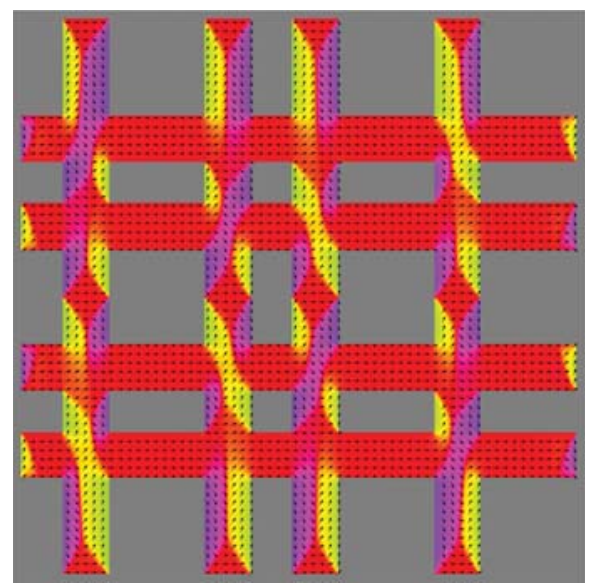

FIG. 3. Simulated magnetization distribution for sample with $W=510 \mathrm{~nm}$. This is in the remnant state after field saturation along the $\mathbf{x}$-direction. DWs are found in both segments and vertices, indicating we are above the Ising regime. 


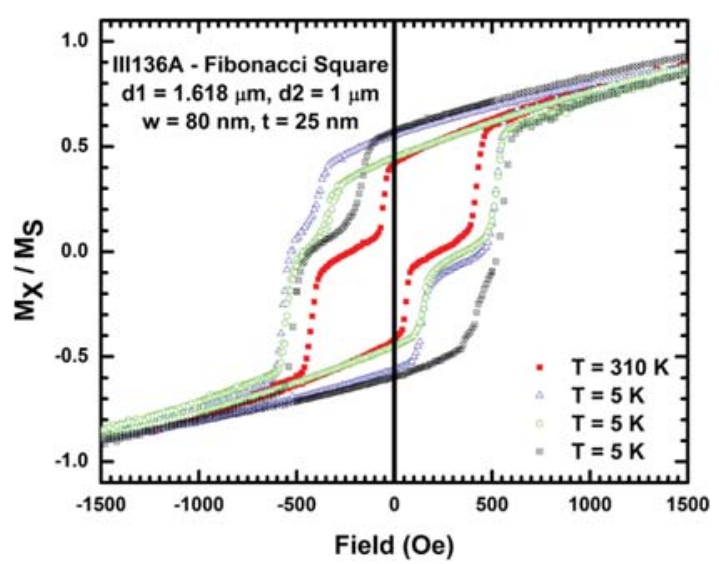

FIG. 4. Horizontal magnetization $\mathrm{M}_{\mathrm{x}}$ normalized to saturation value $\mathrm{M}_{\mathrm{S}}$ versus applied DC field for sample III136A at various temperatures $\mathrm{T}$ shown. Notice the asymmetry with respect to field for the $\mathrm{T}=5 \mathrm{~K}$ data. The sample was positively saturated at $\mathrm{H}=50 \mathrm{kOe}$, then returned to $\mathrm{H}=0 \mathrm{Oe}$ at $\mathrm{T}=300 \mathrm{~K}$, then zero-field-cooled to $\mathrm{T}=5 \mathrm{~K}$, where the sample was again saturated at $\mathrm{H}=50 \mathrm{kOe}$. Blue data were taken during a slow sweep from +50 to -50 to +50 to $0 \mathrm{kOe}$. The green data were taken in a reversed field sequence, -50 to +50 to -50 to +50 to -50 to $0 \mathrm{kOe}$ at $\mathrm{T}=5 \mathrm{~K}$. After the sample was returned to $\mathrm{H}=0 \mathrm{Oe}$, it was heated to $\mathrm{T}=310 \mathrm{~K}$, and the red data were taken, which were symmetric about $\mathrm{H}=0$ Oe. For the black data, the sample was initially placed in $\mathrm{H}=-50 \mathrm{kOe}$ (negatively saturated) at $\mathrm{T}=310 \mathrm{~K}$ then cooled to $\mathrm{T}=5 \mathrm{~K}$, where the protocol for the blue data was repeated in reverse.

antiferromagnetic layer (e.g., $\mathrm{NiO}$ ) is in intimate contact with a soft FM layer (e.g., Py). It is likely that the large surface-to-volume ratio of the Py wire segments shown in Fig. 1(a) enables a Ni-rich surface oxide layer to act as a hard antiferromagnetic bias on the bulk, soft Py segment switching.

Figure 5 shows FMR absorption derivative data for a SFL sample III135B with $W=510 \mathrm{~nm}$. The $9.7 \mathrm{GHz}$ data represent a largely saturated regime in which we observed four or more modes on both sides of zero applied field. Overall, the FMR modes exhibit hysteresis at very low fields $H \leq H_{C}$, but are highly symmetric and reproducible, which suggests the DW structure evolves reproducibly with applied field outside the magnetic reversal regime.

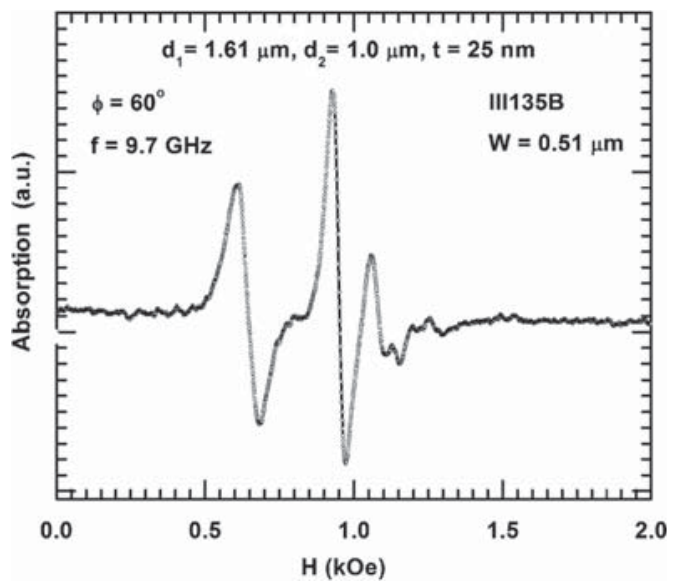

FIG. 5. FMR absorption derivative versus applied field $H$ for sample III135B having segment width $W=510 \mathrm{~nm}$. DC field was aligned at an angle $\phi=60^{\circ}$ with respect to the $\mathrm{SFL}^{10}$ direction. At least four prominent modes are visible in a negative field sweep from $H=12 \mathrm{kOe}$.

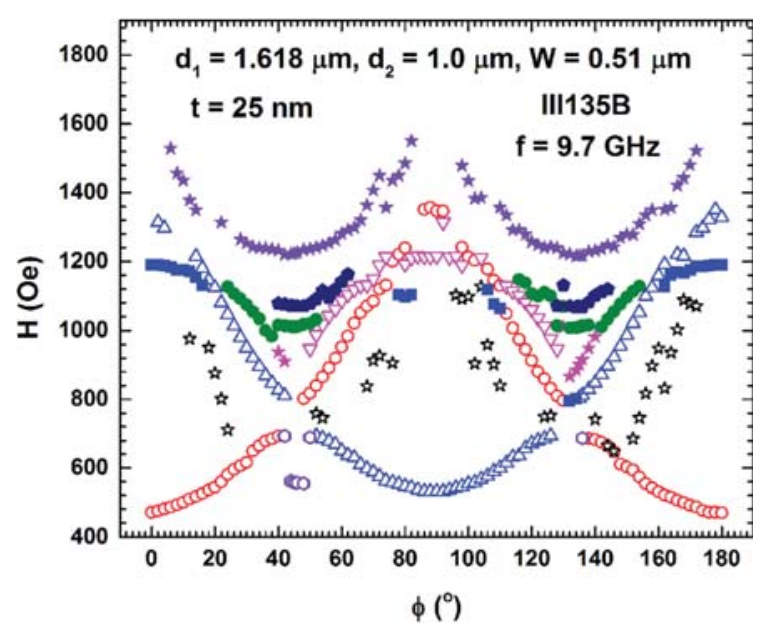

FIG. 6. FMR resonance fields versus angle $\phi$ between the applied field $\boldsymbol{H}$ and the direction ${ }^{10}$ of sample III135B. At least four prominent modes are visible with twofold symmetry; but the spectra collectively exhibit fourfold symmetry. Colored data points indicate assignments to particular modes that are assumed to cross (rather than repel) one another.

Figure 6 shows the variations of the resonance fields on applied field direction for several FMR modes observed for sample III135B. Assuming several modes cross one another, they separately exhibit twofold rotational symmetry, whereas the overall plot obeys fourfold symmetry. This angular symmetry is in accord with the strong shape anisotropy of Isinglike segments in applied fields outside the reversal regime, and with the "average" fourfold symmetry of the aperiodic SFL (i.e., the equal numbers of long and short segments aligned parallel to either the vertical or horizontal SFL directions). ${ }^{9,12}$

As anticipated, FMR spectra and magnetization curves for SFL are more complex than comparable results for similar periodic square permalloy ADL.,15 Additional broadband FMR and numerical simulation studies are underway to investigate the origins of these differences.

Research at the University of Kentucky was supported by U.S. DoE Grant No. DE-FG02-97ER45653, the UK Center for Advanced Materials (supported by U.S. NSF Grant No. EPS0814194), the UK Center for Computational Sciences, and the UK Center for Nanoscience and Engineering.

${ }^{1}$ L. Torres, L. Lopez-Diaz, and J. Iniguez, Appl. Phys. Lett. 73, 3766 (1998).

${ }^{2}$ I. Guedes et al., Phys. Rev. B 62, 11719 (2000).

${ }^{3}$ V. Bhat et al., Physica C 479, 83-87 (2012).

${ }^{4}$ J. Sklenar et al., Appl. Phys. Lett. 102, 152412 (2013).

${ }^{5}$ C. Nisoli, R. Moessner, and P. Schiffer, Rev. Mod. Phys. 85, 1473 (2013).

${ }^{6}$ S. Jung, B. Watkins, L. De Long, J. B. Ketterson, and V. Chandrasekhar, Phys. Rev. B 66, 132401 (2002).

${ }^{7}$ W. Xu et al., J. Appl. Phys. 95(Pt. 2), 6645-6647 (2004).

${ }^{8}$ V. S. Bhat et al., Physica C 503, 170-174 (2014).

${ }^{9}$ V. S. Bhat et al., Phys. Rev. Lett. 111, 077201 (2013).

${ }^{10} \mathrm{~B}$. Grunbaum, Tilings and Patterns (W.H. Freeman, 1990).

${ }^{11}$ E. Y. Vedmedenko, H. P. Oepen, and J. Kirschner, Phys. Rev. Lett. 90, 137203 (2003).

${ }^{12}$ R. Lifshitz, J. Alloys Compd. 342, 186-190 (2002).

${ }^{13}$ D. Levine and P. J. Steinhardt, Phys. Rev. B 34, 596 (1986).

${ }^{14}$ M. J. Donahue and D. G. Porter, OOMMF User's Guide (NIST, 1999).

${ }^{15}$ M. Yu, L. Malkinski, L. Spinu, W. Shou, and S. Whittenburg, J. Appl. Phys. 101, 09F501 (2007). 